UDC 514.74

\title{
INTERPOLATION AND APPROXIMATION BY RATIONAL CURVES OF BEZIER AND NURBS-CURVES
}

\author{
Badayev Yu.I., Dr. Sci. \\ Gannoshina I.M., Art. Teacher, \\ Lagodina L.P., Cand.Tech.Sci, \\ National Technical University of Ukrane "KPI name Igor Sikorsky"
}

Relevance. Rational Bezier curves and NURBS curves are widely used in modeling curvilinear objects due to the great flexibility and efficiency of the method. Therefore, it is relevant to develop an interpolation method and approximation by these curves of a discrete series of points both in the plane and in three-dimensional space.

Method. The work is devoted to the development of a new approach to interpolation and approximations curve fitting, represented by a set of discrete points. The analytical description of the desired curve is implemented using a rational Bezier curve and a NURBS-curve. To solve this problem, two approaches are proposed. The first approach is that the weights of the points are set in advance and then the coordinates of the points of the interpolating or approximating rational Bezier curve as well as the NURBS-curve are calculated. The second approach is that the coordinates of the points are set in advance and then the weights of the control points of the Bezier curve as well as the NURBS-curve are calculated. At the beginning of the process, are set not only coordinates, but also parameters are set to a discrete row of points, that is, each point has the following definition: $T(x, y, u)$ on the plane or $T(x, y, z, u)$ in the three-dimensional space, where $u$-parameter. To solve the interpolation problem, a system of linear equations is created in which each equation reflects the equality between the analytical formula for a curve and a given point. Moreover, the number of interpolated points cannot be more than the order of the interpolating curve. Thus, we have a system of $N$ linear equations, where $N$ is the number of points equal to the number of points of the curve. Unknown are N control points of the desired curve. Moreover, in the first approach, the unknowns are coordinates of control points, and in the second weights of points.To solve the approximation problem, the Least Squares method is used. In the beginning, a sum of squared functional of the terms of the differences between the analytic formula of the curve and the coordinate of the given point is created. The optimization problem of minimizing this functional is solved. For this, a system of linear equations is created., each equation of which is a derivative of the functional with respect to a given parameter and equated to zero. In the first approach, the 
desired parameters are the coordinates of points, and in the second weights of points.

Results.Two methods of interpolation and approximation of a point series by rational Bezier curves and NURBS-curves were developed.

Conclusions. The test cases carried out using computer programs and visualization of results confirm the validity of the proposed methods.

Keywords: interpolation, approximation, rational Bezier curves, NURBS-curves.

Formulation of the problem. To date, when constructing curves in various automation systems, rational Bezier curves and NURBS curves are often used. It is a very flexible tool that allows you to create smooth splines of any order, shapes, and also easy to carry out local control over the curve. The curve is represented in a parametric form and for controlling the shape of the curve using control points and weight coefficients of nodes [1]. Rational nonuniform Bezier curves refer to NURBS and are based on Bernstein's basic functions. The practical application of NURBS curves is very diverse, for example: they are often used in computer graphics to draw smooth curves that accurately describe the shape of the two-dimensional objects depicted in drawings, for the task of the plane curve of building the surfaces of rotation, as well as the simulation of the trajectories of motion on surface and space in the course of time. The parametric representation of the curve allows it to be used in multidimensional spaces. Interpolation and approximation of these curves enables them to be used in modeling objects with complex geometric shapes.

Analysis of recent publications. In work [1] on page 135 offers an algorithm for interpolation with NURBS curves, which is based on the fact that control points in the second stage are projected onto a curve and these new points are taken as new control points. In practice, such an algorithm is difficult to implement and achieves precise results. In the works [2] and [3] It does not specify how to calculate the weight of control points, which prevents the construction of an algorithm for interpolation.

The purpose of the article. The purpose of the article is to develop real algorithms for interpolation and approximation with rational Bezier curves and NURBS curves for a given point series

Main part. This article uses the subroutine NURBS, based on the basic functions of the curves Beyier [1]. When forming Bezier curves, Bernstein's basic functions are used:

$$
B_{i}^{n}(u)=C_{i}^{n} u^{i}(1-u)^{n-i},
$$

where $C_{i}^{n}=\frac{n !}{i !(n-i) !}$ - a binomial coefficient, also called a formula for calculating combinations in combinatorics. The values of the basic function 
(1) are calculated for [1]:

$$
r(u)=\frac{\sum_{i=0}^{N} p_{i} \omega_{i} B_{i}^{N}(\mathrm{u})}{\sum_{i=0}^{N} \omega_{i} B_{i}^{N}(u)},
$$

where $p_{i}$ - the control points of the curve, $\omega_{i}$ - control point weight.

The NURBS- curve is determined by the formula:

$$
r(u)=\frac{\sum_{i=0}^{N} p_{i} w_{i} N_{i k}(u)}{\sum_{i=0}^{N} w_{i} N_{i k}(u)},
$$

where $p_{i}$ - the control points of the curve,

$\omega_{i}$ - control point weight;

$N_{i k}(u)$ - normalized basic power functions degrees k.

Let given point series $R_{j}, j=1,2,3, \ldots K$. Acceptable for each point specific parameters $u_{j}$. We will have a point series $R_{j}\left(x_{j}, y_{j}, u_{j}\right)$. We will interpolate the given point series of the curve (2). To do this we will create an equation system:

$$
\sum_{j=1}^{K}\left[r(u)-\mathrm{R}_{j}\right]=0 \cdot \mathrm{j}=1,2 \ldots \mathrm{K} .
$$

We substitute (2) in (5). We get it:

$$
\sum_{j=1}^{K}\left[=\frac{\sum_{i=0}^{K-1} p_{i} \omega_{i} B_{i}^{N}\left(u_{j}\right)}{\sum_{i=0}^{K-1} \omega_{i} B_{i}^{N}\left(u_{j}\right)}-\mathrm{R}_{j}\right]=0 .
$$

Let's turn (6). We get it:

$$
\sum_{j=1}^{K}\left[\sum_{i=0}^{K-1} p_{i} \omega_{i} B_{i}^{N}\left(u_{j}\right)-\mathrm{R}_{j} \sum_{i=0}^{K-1} \omega_{i} B_{i}^{N}\left(u_{j}\right)\right]=0 .
$$

As you can see, the number of interpolated points should not exceed the number of control points of the curve. Analyzing (6), we can see that the coordinates of the control points can be calculated for interpolation $p_{i}$, if we first assign the value of the weights $\omega_{i}$. You can also calculate the interpolation by calculating the weights $\omega_{i}$, if you assign the coordinates of the control points first $p_{i}$.

As an example, we take the rational Bezier curve of the 3rd degree 


$$
\begin{gathered}
p_{0} \omega_{0}(1-u)^{3}+3 p_{1} \omega_{1}(1-u)^{2} u \\
r(u)=\frac{+3 p_{2} \omega_{2}(1-u) u^{2}+p_{3} \omega_{3} u^{3}}{\omega_{0}(1-u)^{3}+3 \omega_{1}(1-u)^{2} u+3 \omega_{2}(1-u) u^{2}+\omega_{3} u^{3}} .
\end{gathered}
$$

The number of interpolated points must not exceed 4 .

Let's take the next 4-point series with coordinates $\mathrm{x}$, $\mathrm{y}$ and with given parameters u:

$$
\left.\begin{array}{l}
R_{1}\left(x_{1}, y_{1}, u_{1}\right)=R_{1}(8.6802,15.2284,1.0), \\
\mathrm{R}_{2}=R_{2}(13.8645,21.5139,2.0), \\
\mathrm{R}_{3}=R_{3}(17.7323,23.4201,3.0), \\
\mathrm{R}_{4}=R_{4}(21.6309,20.6009,1.0) .
\end{array}\right\}
$$

We substitute (8) into (7). We get a system of 4 linear equations

$\sum_{j=1}^{4}\left[\left(p_{0} \omega_{0}\left(1-u_{j}\right)^{3}+3 p_{1} \omega_{1}\left(1-u_{j}\right)^{2} u_{j}+3 p_{2} \omega_{2}\left(1-u_{j}\right) u_{j}^{2}+p_{3} \omega_{3} u_{j}^{3}\right)-R_{j} W_{j}\right]=0$,

where

$$
W_{j}=\omega_{0}\left(1-u_{j}\right)^{3}+3 \omega_{1}\left(1-u_{j}\right)^{2} u_{j}+3 \omega_{2}\left(1-u_{j}\right) \mathrm{u}_{j}^{2}+\omega_{3} u_{j}^{3} .
$$

We set the weight of control points:

$$
\omega_{0}=1.0, \omega_{1}=2.0, \omega_{2}=3.0, \omega_{3}=1.0 .
$$

Having solved the system (9) separately for $\mathrm{x}$ and $\mathrm{y}$, we will have

$$
\left.\begin{array}{l}
p_{0}\left(x_{0}, y_{0}\right)=p_{0}(0.0,0.0), \\
\mathrm{p}_{1}=p_{1}(10.0,20.0), \\
p_{2}=p_{2}(20.0,30.0), \\
p_{3}=p_{3}(30.0,0.0) .
\end{array}\right\}
$$

The results are obtained using the AutoLISP computer program in the AutoCAD system environment. The test example is shown in Fig. 1.

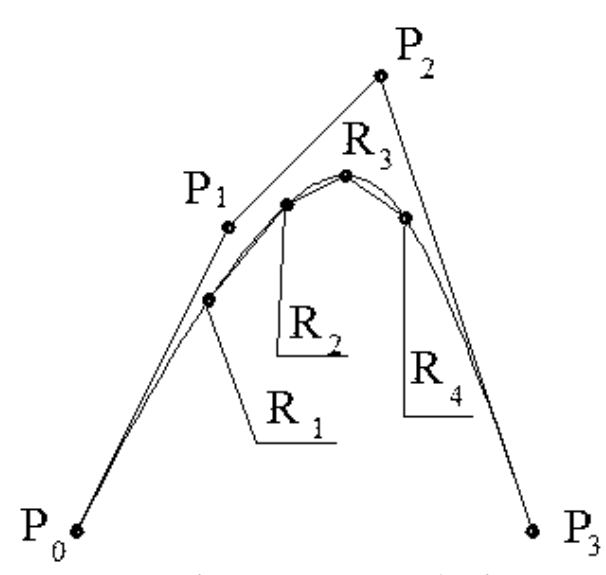

Fig.1. Interpolation

Now, we will make an interpolation by calculating the weighting points of control points. To do this, we rewrite the formula (9) as follows 


$$
\begin{aligned}
& \quad \sum_{j=1}^{4}\left[\left(p_{0}-R_{j}\right) \omega_{0}\left(1-u_{j}\right)^{3}+3\left(p_{1}-R_{j}\right) \omega_{1}\left(1-u_{j}\right)^{2} u_{j}+3\left(p_{2}-R_{j}\right) \omega_{2}\left(1-u_{j}\right) u_{j}^{2}+\right. \\
& \left.\left.+\left(p_{3}-R_{j}\right) \omega_{3} u_{j}^{3}\right)\right]=0, .
\end{aligned}
$$

Unfortunately, system (10) has no solution, so all equations are zero. In order for the system (10) to have a solution, we will accept it $\omega_{0}=1.0$. This is possible because if in equation (8) the numerator and denominator are divided into $\omega_{0}$ то

$$
\omega_{0}=1.0, \bar{\omega}_{1}=\frac{\omega_{1}}{\omega_{0}}, \bar{\omega}_{2}=\frac{\omega_{2}}{\omega_{0}}, \bar{\omega}_{3}=\frac{\omega_{3}}{\omega_{0}} .
$$

Then (10) will be rewritten in the following form

$$
\begin{gathered}
\sum_{j=1}^{4}\left[\left(p_{0}-R_{j}\right)\left(1-u_{j}\right)^{3}+3\left(p_{1}-R_{j}\right) \bar{\omega}_{1}\left(1-u_{j}\right)^{2} u_{j}+\right. \\
\left.\left.+3\left(p_{2}-R_{j}\right) \bar{\omega}_{2}\left(1-u_{j}\right) u_{j}^{2}+\left(p_{3}-R_{j}\right) \bar{\omega}_{3} u_{j}^{3}\right)\right]=0 .
\end{gathered}
$$

System (11) has 4 linear equations and 3 unknowns. This system needs to be reduced to 3 equations. For this we subtract the second equation from the first, third from the second, fourth from the third. In this way we obtain a system of 3 equations with 3 unknowns. Also, for calculation (11) a computer program was developed. To control we leave the coordinates of the control points of the curve $(* *)$ as well as the coordinates of the point series $(*)$.

Calculation (11) gives the following weights:

$$
\bar{\omega}_{1}=2.0, \bar{\omega}_{2}=3.0, \bar{\omega}_{3}=1.0 .
$$

Given that $\omega_{0}=1.0$ we have the same weight points as in the previous version.

Let us consider the possibility of approximation by Bezier's rational curves. To do this, we will apply the known The least squares method. Consider the functional:

$$
S=\sum_{j=1}^{K}\left[r\left(u_{j}\right)-\mathrm{R}_{j}\right]^{2} \Rightarrow \min .
$$

In our case (12) will be rewritten in the form: 


$$
\begin{aligned}
& \begin{array}{l}
p_{0} \omega_{0}\left(1-u_{j}\right)^{3}+3 p_{1} \omega_{1}\left(1-u_{j}\right)^{2} u \\
S=\sum_{j=1}^{4}\left[\frac{+3 p_{2} \omega_{2}\left(1-u_{j}\right) u_{j}^{2}+p_{3} \omega_{3} u_{j}^{3}}{\omega_{0}\left(1-u_{j}\right)^{3}+3 \omega_{1}\left(1-u_{j}\right)^{2} u}\right. \\
+3 \omega_{2}\left(1-u_{j}\right) u^{2}+\omega_{3} u_{j}^{3}
\end{array} \\
& \left.-R_{j}\right]^{2} \Rightarrow \operatorname{min.}
\end{aligned}
$$

or

$$
\begin{aligned}
& \sum_{j=1}^{4}\left[\left(p_{0} \omega_{0}\left(1-u_{j}\right)_{2} \omega_{2}\left(1-3 p_{j}\right) u_{j} \omega_{1}\left(1-p_{3} \omega_{3} u_{j}^{3}\right)\right.\right. \\
& \left.-R_{j} W_{j}\right]_{j} \Rightarrow \min
\end{aligned}
$$

where

$$
\begin{aligned}
& W_{j}=\omega_{0}\left(1-u_{j}\right)^{3}+3 \omega_{1}\left(1-u_{j}\right)^{2} u_{j} \\
& +3 \omega_{2}\left(1-u_{j}\right) u_{j}^{2}+\omega_{3} u_{j}^{3} .
\end{aligned}
$$

Decision (13) will be

$$
\begin{gathered}
\frac{d S}{d p_{0}}=\sum_{j=1}^{4} 2\left[\left(p_{0} \omega_{0}\left(1-u_{j}\right)^{3}+3 p_{1} \omega_{1}\left(1-u_{j}\right) u_{j}^{2}+p_{3} \omega_{3} u_{j}^{3}\right)\right. \\
\left.-R_{j} W_{j}\right] \omega_{0}\left(1-u_{j}\right)^{2}=0 \\
\frac{d S}{d p_{1}}=\sum_{j=1}^{4} 2\left[\left(p_{0} \omega_{0}\left(1-u_{j}\right)^{3}+3 p_{1} \omega_{1}\left(1-u_{j}\right) u_{j}^{2}+p_{3} \omega_{3} u_{j}^{3}\right)\right. \\
\left.-R_{j} W_{j}\right]\left[3 \omega_{1}\left(1-u_{j}\right)^{2} u_{j}\right]=0, \\
\frac{d S}{d p_{2}}=\sum_{j=1}^{4} 2\left[\left(p_{0} \omega_{0}\left(1-u_{j}\right)^{3}+3 \omega_{2}\left(1-u_{j}\right) u_{j}^{2}\left(1-p_{3} \omega_{3} u_{j}^{3}\right)\right.\right. \\
-R_{j} W_{j}{ }^{2} u_{j}\left[3 \omega_{2}\left(1-u_{j}\right) u_{j}^{2}=0,\right. \\
\frac{d S}{d p_{3}}=\sum_{j=1}^{4} 2\left[\left(p_{0} \omega_{0}\left(1-u_{j}\right)^{3}+3 p_{1} \omega_{1}\left(1-u_{j}\right)^{2} u_{j}+3 p_{2} \omega_{2}\left(1-u_{j}\right) u_{j}^{2}+p_{3} \omega_{3} u_{j}^{3}\right)-R_{j} W_{j}\right] \omega_{3} u_{j}^{3}=0,
\end{gathered}
$$

After the differentiation we get a system of 4-line equations:

$$
\left.\begin{array}{l}
a_{0} p_{0}+a_{1} p_{1}+a_{2} p_{2}+a_{3} p_{3}=q_{0}, \\
b_{0} p_{0}+b_{1} p_{1}+b_{2} p_{2}+b_{3} p_{3}=q_{1}, \\
c_{0} p_{0}+c_{1} p_{1}+c_{2} p_{2}+c_{3} p_{3}=q_{2}, \\
d_{0} p_{0}+d_{1} p_{1}+d_{2} p_{2}+d_{3} p_{3}=q_{3} .
\end{array}\right\}
$$

where 


$$
\begin{aligned}
& a_{0}=\sum_{j=1}^{4} \omega_{0}^{2}\left(1-u_{j}\right)^{6}, \quad c_{0}=\sum_{j=1}^{4} 3 \omega_{0} \omega_{1}\left(1-u_{j}\right)^{4} u_{j}^{2}, \quad q_{0}=\sum_{j=1}^{4} R_{j} W_{j} \omega_{0}\left(1-u_{j}\right)^{3}, \\
& a_{1}=\sum_{j=1}^{4} 3 \omega_{0} \omega_{1}\left(1-u_{j}\right)^{5} u_{j}, \quad c_{1}=\sum_{j=1}^{4} 3 \omega_{1} \omega_{2}\left(1-u_{j}\right)^{3} u_{j}^{3}, \quad q_{1}=\sum_{j=1}^{4} R_{j} W_{j}\left[3 \omega_{1}\left(1-u_{j}\right)^{2} u_{j}\right], \\
& a_{2}=\sum_{j=1}^{4} 3 \omega_{0} \omega_{2}\left(1-u_{j}\right)^{4} u_{j}^{2}, \quad c_{2}=\sum_{j=1}^{4} 3 \omega_{2}^{2}\left(1-u_{j}\right)^{2} u_{j}^{4}, \quad q_{2}=\sum_{j=1}^{4} R_{j} W_{j}\left[3 \omega_{2}\left(1-u_{j}\right) u_{j}^{2},\right. \\
& a_{3}=\sum_{j=1}^{4} \omega_{0} \omega_{3}\left(1-u_{j}\right)^{3} u_{j}^{3}, \quad c_{3}=\sum_{j=1}^{4} 3 \omega_{2} \omega_{3}\left(1-u_{j}\right) u_{j}^{5}, \quad q_{3}=\sum_{j=1}^{4} R_{j} W_{j} \omega_{3} u_{j}^{3} . \\
& b_{0}=\sum_{j=1}^{4} 3 \omega_{0} \omega_{1}\left(1-u_{j}\right)^{5} u_{j}, \quad d_{0}=\sum_{j=1}^{4} \omega_{0} \omega_{3}\left(1-u_{j}\right)^{3} u_{j}^{3} \text {, } \\
& b_{1}=\sum_{j=1}^{4} 3 \omega_{1}^{2}\left(1-u_{j}\right)^{4} u_{j}^{2}, \quad d_{1}=\sum_{j=1}^{4} \omega_{1} \omega_{3}\left(1-u_{j}\right)^{2} u_{j}^{3}, \\
& b_{2}=\sum_{j=1}^{4} 3 \omega_{1} \omega_{2}\left(1-u_{j}\right)^{3} u_{j}^{3}, \quad d_{2}=\sum_{j=1}^{4} \omega_{2} \omega_{3}\left(1-u_{j}\right)^{3} u_{j}^{3} \text {, } \\
& b_{3}=\sum_{j=1}^{4} 3 \omega_{1} \omega_{3}\left(1-u_{j}\right)^{2} u_{j}^{4}, \quad d_{3}=\sum_{j=1}^{4} \omega_{3}^{2} u_{j}^{6} .
\end{aligned}
$$

As a result, we calculate the computer program result:

$$
\begin{aligned}
& p_{0}(0.00 .0) ; \\
& p_{1}(9.819 .8) ; \\
& p_{2}(19.9931 .5) ; \\
& p_{3}(30.00 .0) \text {. }
\end{aligned}
$$

As we see, the results of approximation are not much different from interpolation.

A test case is presented in Fig.2. You can also make approximation using weights $\omega_{i}$ control points.

Let's rewrite the functional (13) as follows:

$$
\begin{aligned}
& \sum_{j=1}^{4}\left[\left(p_{0}-R_{j}\right) \omega_{0}\left(1-u_{j}\right)^{3}+3\left(p_{1}-R_{j}\right) \omega_{1}\left(1-u_{j}\right)^{2} u_{j}+\right. \\
& \left.\left.+3\left(p_{2}-R_{j}\right) \omega_{2}\left(1-u_{j}\right) u_{j}^{2}+\left(p_{3}-R_{j}\right) \omega_{3} u_{j}^{3}\right)\right]^{2} \Rightarrow \text { min, }
\end{aligned}
$$

After the differentiation where we get a $\omega_{0}=1.0$.

$$
\begin{aligned}
& \left.\frac{d S}{d \omega_{1}}=\sum_{j=1}^{4}\left[\left(p_{0}-R_{j}\right) \omega_{0}\left(1-u_{j}\right)^{3}+3\left(p_{1}-R_{j}\right) \omega_{1}\left(1-u_{j}\right)^{2} u_{j}+3\left(p_{2}-R_{j}\right) \omega_{2}\left(1-u_{j}\right) u_{j}^{2}+\left(p_{3}-R_{j}\right) \omega_{3} u_{j}^{3}\right)\right] \mathrm{x} \\
& 3\left(p_{1}-R_{j}\right)\left(1-u_{j}\right)^{2} u_{j}=0, \\
& \left.\frac{d S}{d \omega_{2}}=\sum_{j=1}^{4}\left[\left(p_{0}-R_{j}\right) \omega_{0}\left(1-u_{j}\right)^{3}+3\left(p_{1}-R_{j}\right) \omega_{1}\left(1-u_{j}\right)^{2} u_{j}+3\left(p_{2}-R_{j}\right) \omega_{2}\left(1-u_{j}\right) u_{j}^{2}+\left(p_{3}-R_{j}\right) \omega_{3} u_{j}^{3}\right)\right] \mathrm{x} \\
& 3\left(p_{2}-R_{j}\right)\left(1-u_{j}\right) u_{j}^{2}=0,
\end{aligned}
$$


$\left.\frac{d S}{d \omega_{3}}=\sum_{j=1}^{4}\left[\left(p_{0}-R_{j}\right) \omega_{0}\left(1-u_{j}\right)^{3}+3\left(p_{1}-R_{j}\right) \omega_{1}\left(1-u_{j}\right)^{2} u_{j}+3\left(p_{2}-R_{j}\right) \omega_{2}\left(1-u_{j}\right) u_{j}^{2}+\left(p_{3}-R_{j}\right) \omega_{3} u_{j}^{3}\right)\right] \mathrm{x}$
$\left(p_{3}-R_{j}\right) u_{j}^{3}=0$,

As a result of computer realization we get the following results $\omega_{0}=1.0, \omega_{1}=2.2, \omega_{2}=3.2, \omega_{3}=0.9$; As you can see, the results are slightly different from the previous ones, so the curve does not exactly pass through the given points (see Fig. 3) The results obtained can also be applied to NURBS curves, just instead of Bernstein's functions $B_{i}^{N}(u)$ it is necessary to take normalized basic functions $N_{i k}(u)$.

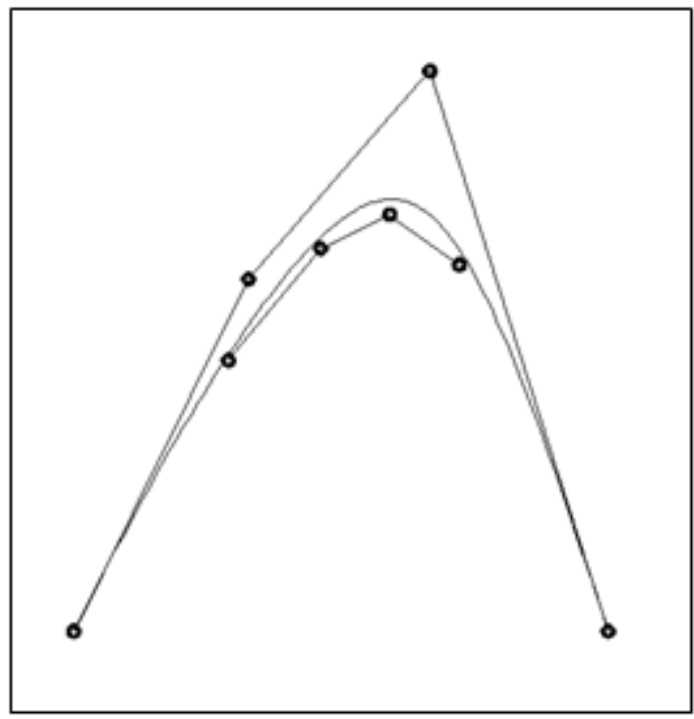

Fig. 2. Approximation

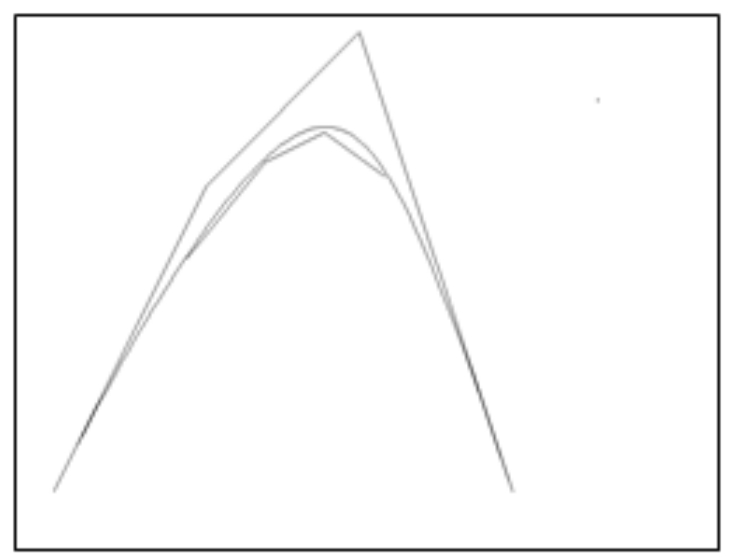

Fig. 3. Approximation using weights of control points

Results. Two methods of interpolation and approximation of a point series by rational Bezier curves and NURBS-curves were developed.

Conclusions. The test cases carried out using computer programs and visualization of results confirm the validity of the proposed methods.

\section{Referenses}

1. Голованов. Н.Н. Геометрическое моделирование. М.: Физматлит, 2002. $472 \mathrm{c}$.

2. Юдин O.A. Интерполяция NURBS-кривыми в многомерном пространстве. Наукові праиі ВНТУ, 2008. № 4. С.1-4.

3. Юдин О.А. Расширение интерполяции по Лагранжу с использованием кривых Безье : Нові технології, 2005. №3(9). C.117-120.

4. David F. Rogers, Rae A. Earnshaw (editors), "State of the Art in Computer Graphics - Visualization and Modeling", 1991, New York, Springer-Verlag, P. $225-269$ 
5. Кветний Р.Н. Методи комп'ютерних обчислень. Вінниця: ВДТУ, 2001. 148 c.

6. Гергель В.П. Теория и практика параллельных вычислений. М: Бином. Лаборатория знаний, 2007. 423 с.

7. Погорелов А. В. Геометрия. М. : Наука, Физматгиз, 1983. 288 с.

8. Фокс А. Пратт М. Вычислительная геометрия. Применение в проектировании и на производстве : пер. с англ. М. : Мир, 1982. $304 \mathrm{c}$.

9. Бадаєв Ю. І., Блиндарук А. О. Керування кривиною NURBS-кривої 3-го порядку за допомогою ваги контрольних вектор-точок. Водний транспорт : зб. наук. прачь Київської державної академії водного транспорту. 2014. № 3 (21). C. 103-105. URL: http://nbuv.gov.ua/UJRN/Vodt_2014_3_21.

10. Бадаєв Ю. І., Блиндарук А. О. Можливості локальної модифікації гладкої NURBS-кривої. Современные информационные $u$ электронные технологии: XV Международная научнопрактическая конференция: науч. труды. Одеса, 2014. Т. 1. С. 26 27.

11. Бадасв Ю. І., Блиндарук А. О. Комп’ютерна реалізація проектування криволінійних обводів методом NURBS-технологій вищих порядків. Сучасні проблеми моделювання : зб. наук. пращь / МДПУ. Мелітополь, 2014. С. 3-6.

\title{
ІНТЕРПОЛЯЦІ І АПРОКСИМАЦІЯ РАЦІОНАЛЬНИМИ КРИВИМИ БЕЗЬС I NURBS-КРИВИМИ.
}

\author{
Бадаєв Ю.І., Ганношина І. М., Лагодіна Л.П.
}

Раціональні криві Безье $i$ NURBS-криві широко застосовуються в моделюванні криволінійних об'єктів завдяки великій гнучкості $i$ працездатності метода. Тому актуальним $\epsilon$ розробка методу інтерполячії $i$ апроксимаціï циими кривими дискретного ряду точок як на площині так $i$ в тривимірному npocmopi.

Робота присвячена розробиі нового підходу до інтерполячії $i$ апроксимачії кривої, поданої множиною дискретних точок. Аналітичний опис шуканої кривої реалізується із застосуванням рачіональної кривої Безьє і NURBS-кривої. Для розв'язання иүієї задачі пропонується два підходи.

Перший підхід заключається в тому, щзо заздалегідь задаються ваги контрольних точок $i$ далі розраховуються координати контрольних точок інтерполюючої або апроксимуючої раціональної кривої Безье а також NURBS-кривої. Другий підхід 
заключається в тому, що заздалегідь задаються координати контрольних точок $i$ далі розраховуються ваги контрольних точок кривої Безьє а також NURBS-кривої.

На початку прочесу дискретному ряду точок задаються не тільки координати, але й також параметри, тобто кожна точка має наступне визначення: $T(x, y, u)$ на площині або $T(x, y, z, u)$ в тривимірному просторі, де $и$ - параметр.Для розв'язання задачі інтерполяиії створюється система лінійних рівнянь, в якому кожне рівняння відображає рівність між аналітичною формулою кривої $i$ заданою точкою. При иьому кількість інтерполюємих точок не може бути більше порядку інтерполюючої кривої. Таким чином маємо систему із $N$ лінійних рівнянь, де $N$ - кількість точок, який дорівнює кількості контрольних точок кривої. Невідомими $\epsilon N$ контрольних точок иуканої кривої. Причому в першому підходi шуканими невідомими є координати контрольних точок, а в другому ваги контрольних точок.Для розв'зання задачі апроксимачії застосовується Метод Найменших Квадратів.На початку створюється функиіонал суми квадратів членів розбіжностей між аналіточною формулою кривої $i$ координатою заданої точки. Розв'язується оптимізаційна задача мінімізачї изього функиіонала. Для иьвого створюється система лінійних рівнянь, кожне рівняння якої є похідною функціонала по заданому параметру і прирівненому до нуля. В першому підході шуканими параметрами є координати контрольних точок, а в другому ваги контрольних точок.

Розроблені по два метода інтерполяиії $i$ апроксимації точкового ряду рачіональними кривими Безье і NURBS- кривими.

Проведені тестові приклади за допомогою комп'ютерних програм і візуалізачії результатів підтверджують достовірність запропонованих методів.

Ключові слова: інтерполящія, апроксимаџія, раџіональні криві Безьє, NURBS-криві.

\title{
ІНТЕРПОЛЯЦИЯ И АППРОКСИМАЦИЯ РАЦИОНАЛЬНЫМИ КРИВЫМИ БЕЗЬЕ И NURВS-КРИВЫМИ.
}

\author{
Бадаев Ю.И., Ганношина И. Н., Лагодина Л.П.
}

Работа посвящена разработке нового подхода к интерполяции и аппроксимации кривой, представленной множеством дискретных точек. Аналитическое описание искомой кривой реализуется $c$ применением раџиональной кривой Безье и NURBS-кривой.

Для решения этой задачи предлагается два подхода. Первый подход заключается в том, что заранее задаются веса точек и 
дальше рассчитываются координаты точек интерполирующей или аппроксимирующей раџиональной кривой Безье а также NURBSкривой. Второй подход заключается в том, что заранее задаются координаты точек и дальше рассчитываются веса контрольных точек кривой Безье а также NURBS-кривой.

В начале прочесса дискретному ряду точек задаются не только координаты, но также параметры, то есть каждая точка имеет следующее определение: $T(x, y, u)$ на плоскости или $T(x, y, z, u)$ в трехмерном пространстве, где и - параметр. Для решения задачи интерполяиии создается система линейных уравнений, в котором каждое уравнение отражает равенство между аналитической формуле кривой и заданной точкой. При этом количество интерполируемых точек не может быть больше порядка интерполирующей кривой. Таким образом имеем систему $N$ линейных уравнений, где $N$ - количество точек, равный количеству точек кривой. Неизвестными являются $N$ контрольных точек искомой кривой. Причем в первом подходе искомыми неизвестными являются координаты контрольных точек, а во втором веса точек.

Для решения задачи аппроксимации применяется Метод Наименьших Квадратов. В начале создается функиионал суммь квадратов членов разностей между аналиточеской формулой кривой и координатой заданной точки. Решается оптимизационная задача минимизации этого функиионала. Для этого создается система линейных уравнений, каждое уравнение которой является производной функиионала по заданному параметру и приравненных $к$ нулю. В первом подходе искомыми параметрами являются координаты точек, а во втором веса точек.

Разработань по два метода интерполяции и аппроксимации точечного ряда рачиональными кривыми Безье и NURBS- кривыми.

Проведенные тестовые примеры с помощью компьютерных программ и визуализации результатов подтверждают достоверность предложенных методов.

Ключевые слова: интерполяиия, аппроксимация, раџиональные кривые Безье, NURBS-кривые. 